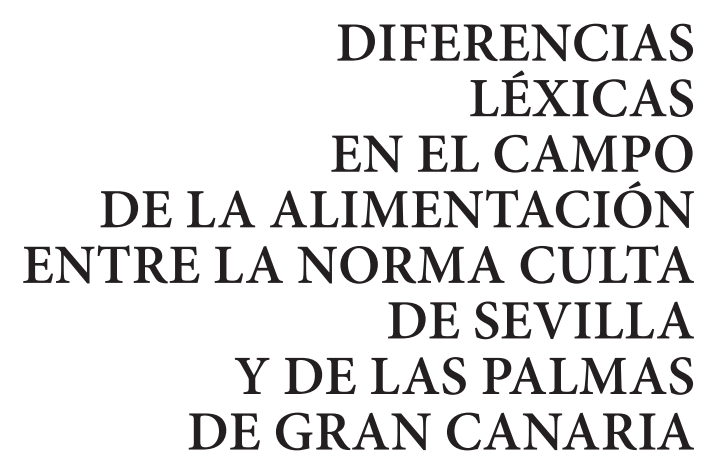


Uno de los objetivos del Proyecto de Estudio de la Norma Culta Hispánica Juan M. Lope Blanch es conocer el léxico activo del sociolecto alto. Para ello se diseñó un cuestionario dividido en 21 campos semánticos y compuesto por 4,452 entradas (PILEI, 1972). Siguiendo dicha metodología común, en este trabajo se hace un análisis contrastivo del área semántica de la alimentación entre las ciudades de Sevilla y de Las Palmas de Gran Canaria. Centramos la atención en las diferencias léxicas, las cuales se distribuyeron en palabras distintas para denominar una misma realidad y palabras iguales para designar conceptos distintos. En el primer grupo, el más numeroso, documentamos unidades léxicas compartidas que presentaban diferencias fónico-gráficas y morfológicas entre una y otra ciudad, así como palabras totalmente distintas para cada zona, donde ocuparon un lugar especial los localismos.

Palabras Clave: norma culta, variación, léxico de la alimentación

One of the aims of the Project for the Study of the Hispanic Educated Norm Juan M. Lope Blanch is knowing the active lexicon in high sociolect. For this purpose, it was designed a questionnaire divided in 21 semantic fields and composed of 4452 items (PILEI, 1972). Following such common methodology, this paper makes a contrastive analysis in the semantic field of food between the cities of Seville and Las Palmas de Gran Canaria. We focused our attention on the lexical differences, which were distributed in distinct words to name the same reality and the same words for different concepts. In the first group, the largest, shared lexical forms with phonic-graphical and morphologic differences between one and another city were found, as well as completely different words for each zone, where localism occupied a special place.

KEY wORDS: educated norm, variation, lexicon of the food 


\section{DIFERENCIAS \\ LÉXICAS \\ EN EL CAMPO \\ DE LA \\ ALIMENTACIÓN \\ ENTRE LA \\ NORMA CULTA \\ DE SEVILLA \\ Y DE LAS PALMAS \\ DE GRAN CANARIA}

Juana Santana

Universidad de Sevilla

Uno de los grandes logros del Proyecto para el Estudio de la Norma Culta Hispánica J. M. Lope Blanch es haber contribuido al conocimiento de los hábitos lingüísticos de los hablantes del sociolecto alto en las principales ciudades del mundo hispánico. ${ }^{1}$ Para ello se propusieron pautas metodológicas comunes que permitieran realizar estudios en cada

\footnotetext{
${ }^{1}$ Las ciudades integrantes en la actualidad son Buenos Aires, Caracas, Ciudad de México, Córdoba-Argentina, La Habana, La Paz, Las Palmas de Gran Canaria, Santiago de Chile y Sevilla.
} 
una de las ciudades integrantes, así como desarrollar análisis contrastivos que pusieran de manifiesto las semejanzas y las diferencias entre las áreas que intervenían en el Proyecto. Para el estudio del nivel léxico-semántico se diseñó un cuestionario de carácter onomasiológico (PILEI, 1972) en el que se partía de un concepto y se le preguntaba al informante por la manera de denominarlo (principalmente a partir de su definición o, en un menor número de veces, mostrando una imagen u objeto). Dicho cuestionario estaba compuesto inicialmente por 4,452 entradas distribuidas en 21 campos semánticos.

El objetivo de esta investigación es comparar los resultados obtenidos en las ciudades de Sevilla [SE] y Las Palmas de Gran Canaria [LPGC] para el campo semántico de la alimentación. Concretamente, nos centraremos en las diferencias léxicas. Si bien las conexiones lingüísticas entre Andalucía y Canarias han sido abordadas en otras investigaciones (Fernández Sevilla, 1981; Moreno Fernández, 1991, etc.), en este trabajo nos movemos dentro de unos límites muy específicos: partimos del contraste de los usos léxicos del sociolecto alto en las ciudades de Sevilla y de Las Palmas de Gran Canaria. Se combinan, por tanto, los intereses de la Dialectología urbana y de la Sociolingüística. Esta investigación complementa a otras realizadas previamente sobre el mismo corpus en el que estudiamos las lexías compartidas (Santana, 2011) y las diferencias ocasionadas por el empleo del diminutivo (Santana, 2014). A su vez, nos sumamos a otras investigaciones llevadas a cabo en el seno del Proyecto de la Norma Culta en las que se realizaron estudios sobre diversos campos semánticos, contrastando los resultados 
de varias ciudades (López Morales, 1992; Luna, 1997, 2005, 2007; Hernández y Samper, 1999, etc.) o los de un núcleo urbano en particular (Valencia, 1984; Salvador, 1992, etc.). En definitiva, nuestro objetivo último es contribuir a la labor conjunta de conocer las preferencias léxicas del sociolecto alto en el mundo hispánico.

\section{La muestra}

Para estudiar el campo de la alimentación partimos de los resultados obtenidos en SE (Carbonero y Ortiz, 2005) y en LPGC (Samper, 1998), donde se pretendía recoger una muestra significativa del léxico activo de los hablantes cultos. Se entrevistó a un total de 12 personas para cada ciudad, todas ellas con estudios universitarios, distribuidas en 3 grupos de edad ( $1^{\text {a }}$ gen. de 25 a 35 años; $2^{\text {a }}$ gen. de 36 a $55 ; 3^{\text {a }}$ gen. de 56 en adelante) y en grupos equilibrados de hombres y mujeres.

Cada uno de los 21 campos semánticos en los que se divide el cuestionario cuenta, a su vez, con un número variado de entradas o conceptos que se distribuyen en secciones. El área de la alimentación está compuesta por 306 entradas, divididas en las siguientes partes: lugares para comer, las comidas, el desayuno, el almuerzo, los huevos, el pescado, carne de cerdo, carne de cordero, carne de res, carne de pavo, las aves, las pastas, el arroz, verduras y hortalizas, legumbres, los condimentos, los postres, formas de preparar los alimentos, bebidas, leche, té, infusiones y lugares para tomar bebidas alcohólicas. 
Aunque los criterios metodológicos seguidos por las ciudades seleccionadas para el estudio son básicamente los mismos, se aprecian algunas mínimas variaciones en el tratamiento de la muestra de SE y de LPGC. ${ }^{2}$ Para evitar desajustes en el número de conceptos estudiados y en los resultados de la investigación, no se han tenido en cuenta aquellas entradas que se vieron afectadas por cambios del tipo aglutinación, omisión o adición de conceptos y que se realizaron solo en los materiales de una de las zonas exploradas: 351 . CHOCOLATE ESPESO, 352. CHOCOLATE A LA FRANCESA, 371. pasapalos, 603. VERMOUTH, 619. CafÉ CON LeCHE y 633. GRILL, PARRILla. Teniendo en cuenta estas reducciones, la cantidad total de conceptos estudiados es de 300 .

\section{Análisis lingüístico}

Las diferencias en el léxico de los hablantes cultos sevillanos y canarios pueden distribuirse en los siguientes bloques:

A) Palabras solo empleadas en una de las ciudades.

B) Palabras empleadas en ambas ciudades pero con sentidos diferentes.

C) Variaciones entre una voz estándar y un localismo con distinta distribución en ambas ciudades.

\footnotetext{
${ }^{2}$ No existe total homogeneidad en las estrategias empleadas para la obtención de las respuestas en las ciudades estudiadas. El material de SE incluye el recurso empleado en cada entrada y, en su caso, la definición utilizada. Sin embargo, la publicación de LPGC no cuenta con esta información, aunque sus editores explican con detalle las decisiones adoptadas en la elaboración de la encuesta y en la posterior publicación del material (Samper 1998: 9-25). También aparece información detallada al respecto en Marrero (1999: 77-100).
} 
El estudio de estos bloques nos permitirá comprobar, entre otros aspectos, la rentabilidad que tienen en la norma culta voces específicas o significaciones particulares que adquieren algunos términos en cada una de estas áreas geográficas, los llamados andalucismos o canarismos léxicos, concretamente los relacionados con el campo de la alimentación. Al mismo tiempo, podremos observar las diferencias léxicas, cuando no son voces de carácter regional, para designar realidades conocidas en ambas áreas urbanas.

Es preciso señalar que la ausencia de una palabra entre las respuestas de uno de los núcleos urbanos no significa que esta no se encuentre entre el léxico activo de sus hablantes. En algunos casos así es, pero en otros, principalmente cuando hay abundante variación sinonímica en las contestaciones, lo que sucedió es que los informantes prefirieron otras opciones léxicas, sin que eso suponga que las que habían descartado y que, a su vez, habían sido utilizadas en la otra ciudad, las desconocieran. A este respecto, seleccionamos aquellas unidades léxicas que aportaron información relevante para observar las diferencias diatópicas entre SE y LPGC. Por ejemplo, no creemos necesario incluir respuestas como agua de poleo y agua de manzanilla, que solo respondieron los informantes canarios, o cava y cítrico que solo respondieron los sevillanos, pues no apor$\tan$ datos relevantes para esta investigación. Teniendo en cuenta estas delimitaciones, nuestro corpus de estudio está compuesto por un total de 130 lexías, distribuidas entre 58 de SE y 72 de LPGC. 
Achicoria-chicoria (570. maLta); alcaucil, alcachofa (489. ALCACHOFA); bacón (446. TOCINO FRITO; 448. TOCINO CON VETA); bodeguita (634. TABERNA); bollito (370. BOCADITOs); brócoles, brócoli (495. BRócOLI); caldito [de carne, de pollo, con huevo, con vino] (401. el CALDo, 402. Consomé, 403. CONSOMÉ DE CARNE, 404. CONSOMÉ DE POLLO, 405. CONSOMÉ CON HUEVO, 406. CONSOMÉ CON VINO); calentitos, churros (356. LOS CHURRos); chícharos [blancos, colorados, con pintas, negros], ${ }^{3}$ judías, alubias, guisantes (501. JUDÍAs BLANCAS; 502. JUDÍAS PINTAS, 503. JUDÍAS NEGRAS, 504. JUDÍAS ROJAS Y 507. GUISANTES, CHÍCHAROS, ARVEJAS); champagne-champán (605. CHAMPAÑA); chile-chili (515. AJÍ, CHILE); chuletitas (424. CHULETAS DE CORDERO); clavo (523. CLAVO); cocido (572. ALIMENTO COCIDO); cogollito (484. COGOLLO); endibia (481. BERRo); garbanzos (508. GARBANZOs); hervido (572. ALIMENTO COCIDO); higaditos (422. ASADURAS, vísCERAS); letritas (467. PASTAS DE LETRAS); maíz (390. SOPA DE MAÍz); manchaíto, manchado (620. LECHE CON UNAS GOTAS DE CAFÉ); mango (540. PAPAYA); manzanilla (364. vino Blanco); [tortilla de] papas, [sopa de] [tortilla de] patatas (389. SOPA DE PAPA y 415. TORTILLA DE PATATAS); papaya (540. PAPAYA); pastelito (565. PASTEL PEQUeÑo); pero, manzana (531. manzANA); picadillo, ensalada (478. LA ENSALADA); piscolabis-piquislabi (341. COMIDA A MEDIA MAÑANA); puerro (481. BERRo); pudin-budín (526. PUDIN);

\footnotetext{
${ }^{3}$ Colocamos entre corchetes el contexto en el que aparece el vocablo que nos interesa para nuestro estudio.
} 
rábano (499. RÁBANO); redonda, redondilla (435. CARNE RELLENA); restaurante (331. RESTAURANTE, 332. RESTAURANTE DE LUJO, 333. RESTAURANTE MEDIANO Y 334. RESTAURANTE MODESTO); romanilla, escarola (483. ESCAROLA); salami (442. SAlAME); tapitas (368. TAPAS); tostada, pan tostado, pan horneado (355. PAN TOSTADO).

\section{LPGC}

Aguachirri (613. CAFÉ MUY DILUIDO); agüita (624. AGUA CALIENTE DE HIERVAS AROMÁTICAs), arvejas, guisantes (507. GUISANTES, CHÍCHAROS, ARVEJAS); barillo, barito (631. LUGARES PARA TOMAR BEBIDAS, DE ÍNFIMA CALIDAD); beicon (445. TOCINO; 447. TOCINO DE PERNIL; 448. TOCINO CON VETA); berro (481. BERRO), beterrada (497. REMOLACHA), bichillo (421. SOLOMILLO); [pan] bizcochado, bizcocho (355. PAN TOSTADO), bochinche, bodeguilla (631. LUGARES PARA TOMAR BEBIDAS, DE ÍNFIMA CALIDAD); bodega (630. BAR, LUGAR EXCLUSIVO PARA TOMAR BEBIDAS); buche de café, cafetito, café corto (616. CAFÉ CORTO); cafetín (630. BAR, LUGAR EXCLUSIVO PARA TOMAR BEBIDAs); caldo [de papas, de millo, de maíz, de arroz, de verduras, de carne, de pescado, de pollo, de gallina] (389. SOPA DE PAPA; 390. SOPA DE MAÍZ; 391. SOPA DE ARROZ; 397. SOPA DE VERDURAS; 398. SOPA DE CARNE; 400. SOPA DE PESCADO; 401. EL CALDO, 402. CONSOMÉ; 404. CONSOMÉ DE POLLO); champán (605. CHAMPAÑA); chicoria (570. MALTA); chile (515. AJÍ, CHILE); chuletas [de cordero] (424. CHULETAS DE CORDERO); cilantro (500. CilANTRO); clavillo (523. ClAVO); cogollo (484. COGOLLO); [pollo] compuesto (456. POLLO CON TOMATE Y PIMIENTO; 
575. Alimento ESTOFAdo; 460. POllo Estofado); conduto (370. Bocaditos); conserva [de membrillo] (549. DULCE MEMBRILlo O CARNE DE MEMBRILlo); dulce (565. PASTEL PEQUEÑo); durazno (533. MELOCOTÓN); enyesques (368. TAPAS; 370. BOCADITOs); garbanzas (508. GARBANZOS); gofio (475. GOFIO); guanijai (345. REFRIGERIO DESPUÉS DE LA CENA); guarapo (613. CAFÉ MUY DILUIDO); higadillos (422. ASADURAS, VÍSCERAS); jarea (416. SALAZÓN, PESCADO SALADO); jugo (347. EL JUGo); letras [para sopa] (467. PASTAS DE LETRAS); [café] manchado (620. LECHE CON UNAS GOTITAS DE CAFÉ); [sopa de, caldo de, potaje de] millo, maíz (390. SOPA DE MAÍz); [caldo de, sopa de] [tortilla de] papas, [sopa de] [tortilla de] patatas (389. SOPA DE PAPA y 415. TORTILLA DE PATATAS); papayo (540. PAPAYA); pastelillo (565. PASTEL PEQUEÑo); pescado salado (416. SALAZÓN, PESCADO SALADO); [alimento] picón (593. AlIMENTO PICANTE); pimienta de la puta la madre (515. AJÍ, CHILE); piscolabis (339. COMIDA ANTES DEL DESAYUNO; 341. COMIDA A MEDIA MAÑANA; 368. TAPAS; 370. BOCADITOS); pizqueo (345. REFRIGERIO DESPUÉS DE LA CENA; 368. TAPAS; 370. BOCADITOS); potaje (397. SOPA DE VERDURAS); pudín (526. PUDIN); quequequequi [de frutas] (564. BIZCOCHO; 566. PASTEL DE FRUTAS; 568. TORTA); rabanillo (499. RÁBANO); restaurán-restaurante (331. ReSTAURANTE, 332. RESTAURANTE DE LUjO, 333. RESTAURANTE MEDIANO y 334. RESTAURANTE MODESTO); rosquillas (361. LAS PASTAS); salame-salami (SALAME); [huevos] sancochado(-s) (408. HUEvos DUROs. 572. ALIMENTO COCIDO; 579. ALIMENTO SANCOCHADO); tabernilla (631. LUGARES PARA TOMAR BEBIDAS, DE ÍNFIMA CALIDAD); $t a-$ pas (368. TAPAs; 370. BOCADITOs); taza de agua (624. AGUA 
CALIENTE DE HIERBAS AROMÁTICAS); tortilla (547. TORRIJA, TORREJA); [pan] tostado (355. PAN TOSTADo); vuelta (437. FILETE); [taza de, agua con] yerbahuerto, [infusión de, agua de] yerbabuena (626. INFUSIÓN DE YERBABUENA).

\subsection{Palabras solo empleadas en una de las ciudades}

La casuística de lexías que constituye este bloque es diversa $\mathrm{y}$ admite nuevas subdivisiones.

2.1.1. El concepto solo es reconocido en una de las ciudades

Si bien la mayoría de los conceptos por los que se preguntó fueron identificados por al menos 1 informante de cada zona, hubo 4 entradas que obtuvieron una respuesta unánime entre los hablantes cultos canarios, frente a la ausencia de contestación (o respuesta distinta a lo que se preguntaba) entre los sevillanos: 475. GOFIO, 481. BERRO, 500. CILANTRO y 579. ALIMENTO SANCOCHADO. No se registró ningún concepto que fuera reconocido solo en SE.

El gofio, 'harina de maíz tostado', es un alimento típico de la cocina canaria, por lo que fue identificado con claridad por todos los encuestados de LPGC. Esta palabra es considerada un canarismo por las obras especializadas (Diccionario ejemplificado de canarismos [DEC], Diccionario básico de canarismos [DBC], Tesoro lexicográfico del español de Canarias [TLEC], Marrero 1996-1997 y 1999), en este caso de origen guanche. Se trata de una lexía que no entra en competencia sinonímica con otras voces. 
En el caso de berro, en SE hubo solo 2 contestaciones, distribuidas en 2 informantes: endibia y puerro. De aquí se deduce que no se identificó con claridad la realidad por la que se preguntaba, porque no es una verdura habitual en las costumbres culinarias sevillanas. Para cilantro, pensamos que por las mismas razones, no hubo ninguna contestación en SE. Esto supone una diferencia clara con respecto a la norma culta canaria, donde las respuestas de todos los encuestados fueron berro y cilantro respectivamente.

En lo que respecta a sancochado, el hablante canario maneja esta denominación para 'alimento que se cocina en agua o al vapor, por lo general sin emplear aceite'. Por eso apareció como respuesta de 572. ALIMENTO COCIDO y de 579. alimento sancochado. Sin embargo, el hablante sevillano solo reconoce cocido y hervido, respuestas que emplea en 572, pero no sancochado, para lo que no hay contestación. Se trata de una palabra que no es habitual en esta ciudad.

\subsubsection{El concepto es reconocido en ambas ciudades}

Este grupo está formado por lexías que fueron respuestas distintas en SE y en LPGC a conceptos claramente identificados y conocidos en ambas zonas. Dentro de la variada casuística podemos hacer varios subgrupos, dependiendo de cuáles sean las razones que explican las diferencias: rasgos fónico-gráficos, rasgos morfológicos y dialectalismos. En ocasiones un término puede ubicarse en más de uno de estos apartados. 


\subsubsection{Diferencias fónico-gráficas}

Un primer bloque está constituido por voces que comparten una misma base léxica pero que presentan diferencias fónicas, característica que ha pasado a la escritura. En esta situación están bacón / beicon; ${ }^{4}$ champagne-champán / champán; chicoria-achicoria / chicoria; chile-chili / chile; piscolabispiquislabi / piscolabis; pudin-budin / pudin; restaurante / restaurante-restaurán; salami / salame-salami.

En los casos de pudin-pudín; beicon-bacón; chicoriaachicoria y salami-salame el DRAE señala (bien en forma de lema compartido o bien remitiéndonos a otro lema) que son posibles ambas representaciones gráficas. No podemos descartar que haya sido decisión de los editores transcribir de una u otra manera. Sin embargo, el hecho de que haya una diferencia fónica significativa en el caso de beicon / bacón, así como el hecho de que para chicoria-achicoria o salamisalame se recogieran las 2 variantes en el léxico culto de SE en el primer caso y de LPGC en el segundo, nos hace pensar que la transcripción es reflejo de lo que pronunciaron los informantes. En lo que respecta a las preferencias por uno de los miembros de estas parejas que se dieron en una misma ciudad, chicoria fue más empleada que achicoria por los hablantes cultos sevillanos $(2 / 3,66.67 \%)$ y los hablantes cultos canarios prefirieron salami en vez de salame (3/4, $75 \%$ ), aunque los usos estuvieron bastante equilibrados y en ambos casos hubo pocas respuestas.

Para las variantes pudín-budín, chile-chili y piscolabispiquislabi, las pronunciaciones no estándares (situadas a la

\footnotetext{
${ }^{4}$ La disposición que seguimos es SE / LPGC.
} 
derecha), no recogidas por la Academia, fueron respuesta de 1 solo informante y, en todos los casos, convivieron con la forma canónica. Nos inclinamos por pensar que fueron usos espontáneos, aislados, quizá debidos al desconocimiento del informante de la verdadera pronunciación de la palabra.

En champagne-champán (alternancia que se dio en SE) y restaurán-restaurante (alternancia que se dio en LPGC) entran en competencia la variante extranjerizante con la adaptación castellana. Champagne fue la forma preferida por los hablantes cultos sevillanos $(6 / 11,54.55 \%)$, principalmente entre los grupos etarios más jóvenes, por lo que se prevé que esta variante se mantenga en esta comunidad de habla. Por su parte, en LPGC la variación restaurán-restaurante puso de manifiesto la preferencia de la segunda, es decir, la forma castellanizada (57/63, 90.48\%). Todos los casos de restaurán fueron empleados por mujeres de la primera y de la segunda generación, lo que hace prever que su uso siga perviviendo en la norma culta de la ciudad, aunque en clara desventaja con la forma con la que compite.

\subsubsection{Diferencias morfológicas}

Uno de los rasgos diferenciadores que hemos observado a partir del análisis del corpus es el uso compartido de bases léxicas que, sin embargo, tienen distinto morfema de género en una y otra ciudad o que presentan diferencias en cuanto al uso del diminutivo.

En la mayoría de los casos, no tuvimos en cuenta las variaciones de género (dentro de una misma ciudad o entre ambas zonas) porque no implicaban diferencias léxicas. 
No obstante, registramos algunos ejemplos donde la alternancia masculino-femenino sí aportó datos sobre los usos léxicos de ambos núcleos urbanos. En esta situación se encuentran garbanzos / garbanzas, papaya / papayo y tostada / [pan] tostado.

Garbanzas y papayo son 2 canarismos que perviven en la norma culta de LPGC. Aunque en el primer caso el DRAE dice que es un 'garbanzo mayor, más blanco y de mejor calidad que el corriente', 1 informante canario (hombre de la tercera generación) lo usa como segunda opción para designar al garbanzo. Como se puede apreciar, su uso no tiene especial repercusión en la norma culta de la capital.

Por su parte, el canarismo papayo, empleado para denominar el 'fruto tropical, de forma oblonga, cuya parte carnosa es parecida el melón', es decir, lo que el DRAE identifica con papaya, fue la variante léxica más relevante en LPGC $(10 / 13,76.92 \%)$. Uno de los informantes que empleó papaya respondió papayo como primera opción. El hecho de que 2 informantes de la primera generación hayan empleado $p a-$ paya en sus respuestas puede ser indicio de que se esté produciendo un proceso de estandarización, aunque todavía no hay una competencia significativa con la denominación característica de la zona. En SE solo se respondió papaya, junto a mango y a la ausencia de respuesta (quizá porque no es un tipo de fruto que entre habitualmente en las costumbres alimenticias de la ciudad andaluza).

Por último, tostada es la denominación preferida por los hablantes cultos sevillanos para el 'pan tostado al fuego antes de comerlo' (10/12, 8.33\%), más usada que otras 2 variantes minoritarias, pan horneado y pan tostado, con 1 sola 
respuesta en cada caso. En LPGC no se utiliza tostada, solo pan tostado y, principalmente, pan bizcochado. El Tesoro léxico de las hablas andaluzas [TLHA] señala que en el Atlas lingüístico y etnográfico de Andalucía [ALEA] quedó registrada tostada en la zona de Huelva para referirse al 'alimento que se toma en el desayuno'. Es, por tanto, una diferencia léxica entre SE y LPGC.

El uso de bizcochado se considera un canarismo y es la variante más empleada en LPGC. Se trata de una muestra de la tendencia a mantener voces arcaicas en esta área dialectal: bizcochado hace referencia a la raíz etimológica bi-coctus ('cocido dos veces para que el pan dure más tiempo'). En el avance de la nueva edición del DRAE se recoge esta acepción, aunque no se le atribuye marca diatópica.

Otro bloque de variaciones morfológicas se debe al uso del diminutivo en las respuestas de una y otra ciudad. Distinguimos los casos en los que se empleó como marca de derivación apreciativa y aquellos en los que se ha lexicalizado.

Los usos del diminutivo como sufijo que documentamos fueron los siguientes:

SE: bodeguita, caldito [de carne, de pollo, con huevo, con vino], chuletitas, cogollito, higaditos, letritas, manchaíto, pastelito y tapitas.

LPGC: barillo, barito, bodeguilla, cafetito, pastelillo y tabernilla.

Una primera conclusión a la que se puede llegar a partir de estos datos es que el diminutivo fue ligeramente más productivo en la norma culta de SE (9/15, 60\%), aunque los porcentajes no marcan diferencias significativas con 
respecto a LPGC. Hay que destacar también que en la norma culta sevillana se utilizó solo la variante en -ito, mientras que en la norma culta canaria la más frecuente fue -illo $(4 / 6,66.67 \%)$.

En la mayoría de los casos el diminutivo empleado en una de las ciudades alternó con la misma base léxica sin derivación en la otra (SE: caldito, chuletitas, cogollito, letritas, manchaito y tapitas / LPGC: barillo, barito, cafetito y tabernilla).

Destacamos el uso de chuletitas en 5 informantes sevillanos $(5 / 12,41.67 \%)$, con bastante vitalidad en esta ciudad, más frecuente que chuletas $(4 / 12,33.33 \%)$, lo que demuestra que se trata de una variante bastante asentada entre los hábitos lingüísticos de la ciudad, frente a su ausencia en LPGC, donde se respondió chuletas de cordero, costillas de cordero y paletillas de cordero.

Manchaito, aunque con poca documentación en SE (solo 1 ejemplo), es una forma frecuente en esta ciudad. Quizá el cuestionario haya frenado su aparición porque suele emplearse en un registro coloquial. Mancha $(d)_{o}$ y manchaito como sustantivos están bastante asentados en la capital andaluza para designar 'la combinación de café con poca cantidad de leche', mientras que en la capital canaria no parece haber una forma léxica específica para su denominación: leche con un poco de café, leche con una nube de café, leche con café, café con leche...

También documentamos la alternancia rábano / rabanillo y clavo / clavillo, donde las formas con diminutivo, con lema independiente en el DRAE, son consideradas variantes sinonímicas de su correspondiente base léxica sin derivación, por lo que el hablante pudo escoger entre una u otra 
opción. Ni para rabanillo ni para clavillo se documentaron respuestas entre los informantes más jóvenes, por lo que son formas que presentan tendencia a la sustitución por la variante más estándar, sin sufijación. Además, rábano y clavo fueron más empleadas tanto en SE como en LPGC.

En consonancia con la distribución de diminutivos -ito / -illo ya señalada, se registró la pareja pastelito / pastelillo, aunque ambas en representación minoritaria con respecto a pastel para SE y, en el caso de LPGC, a dulce, canarismo que presenta una fuerte pervivencia entre los informantes cultos. En higaditos / higadillos observamos la misma distribución de las formas del diminutivo, esta última con lema independiente en el DRAE.

Aunque el escaso número de vocablos registrados no nos permite llegar a conclusiones definitivas, hemos contrastado el empleo del diminutivo en hombres y mujeres para comprobar si fue más empleado por las féminas, como señalaba García Mouton (1999: 72). Los resultados del análisis muestran un ligero predominio del empleo de este sufijo entre las mujeres $(9 / 15,60 \%)$, bien porque fueron ellas las únicas que lo utilizaron (bodeguita, caldito, cogollito, higaditos, pastelito, barito, bodeguilla), o bien porque mostraron un mayor número de respuestas que los hombres (chuletitas, tapitas). Nuestros resultados confirman la característica atribuida a este grupo social de una mayor tendencia al empleo del diminutivo, aunque los porcentajes no son determinantes.

Y un último subgrupo es el de los diminutivos que se han lexicalizado y han pasado a formar parte de nuestro acervo léxico. Aquí nos centramos en aquellas voces que contribuyeron a acentuar las diferencias entre la norma cul- 
ta de ambas ciudades, en tanto que se documentaron solo en una de las zonas y, además, en su mayoría constituyen unidades léxicas con marcas diatópicas. En SE registramos bollitos, calentitos, picadillo, redondilla y romanilla; y en LPGC documentamos agüita, bichillo y rosquillas. En todos los casos, estas formas fueron superadas en respuestas por otras variantes más comunes y estandarizadas, a menudo empleadas en ambas ciudades: canapés en vez de bollitos, churros en lugar de calentitos, ensalada en vez de picadillo, carne rellena por redondilla, escarola en lugar de romanilla, infusión antes que agüita, solomillo en vez de bichillo y pastas en lugar de rosquillas.

También dentro de los diminutivos lexicalizados destacamos el uso compartido de 2 unidades léxicas empleadas en ambas ciudades, pero con significaciones distintas y, además, cada una de ellas relacionada con los hábitos culinarios específicos en su área geográfica. Fueron los casos de manzanilla y tortilla, que se incluyen en el siguiente apartado, en tanto que variantes regionales. ${ }^{5}$

\subsubsection{Dialectalismos}

En ocasiones las diferencias entre las respuestas obtenidas en SE y en LPGC se deben a la pervivencia en una u otra ciudad de variantes léxicas de carácter local que, como era de esperar, no fueron compartidas por la otra área con la que se contrasta. Para delimitar lo que consideramos dialectalismo léxico adoptamos los criterios que emplean

\footnotetext{
${ }^{5}$ Por razones de espacio no podemos desarrollar por extenso las particularidades en el uso del diminutivo en el campo semántico de la alimentación, aspecto que abordamos en Santana (2014).
} 
Corrales y Corbella (2009: 16) para definir canarismo. Se trata de voces específicas de las áreas estudiadas, que no se emplean en otras zonas del ámbito hispánico, o bien de vocablos compartidos con otras zonas pero que en Andalucía o en Canarias adquieren una significación especial o un uso característico.

De forma general, la norma culta de LPGC muestra un mayor uso de canarismos ${ }^{6}(36)$, frente a la menor presencia de andalucismos en SE (9).

SE: alcaucil, bollitos, brócoles, calentitos, chícharos, manzanilla, pero, picadillo, romanilla.

LPGC: ${ }^{7}$ aguachirri, arvejas, beterrada, bichillo, bizcocho, bizcochón, bochinche, buche de café, cafetín, [pollo] compuesto, conduto, conserva, dulce, durazno, enyesques, garbanzas, guanijai, guarapo, jarea, jugo, [sopa de, caldo de, potaje de] millo, [pan] bizcochado, [caldo de, sopa de] [tortilla de] papas, papayo, pescado salado, picón, pimienta de la puta la madre, pizqueo, [huevos] sancochados, queque-quequi, rosquillas, tortilla, taza de agua, vuelta, [taza de, agua con] yerbahuerto.

\footnotetext{
${ }^{6}$ A este respecto cabe señalar que ya Hernández y Samper (2003) demostraron la gran vitalidad que tienen los regionalismos en el léxico disponible de los estudiantes preuniversitarios de LPGC.

${ }^{7}$ La palabra habichuela aparece consignada como canarismo en las obras lexicográficas consultadas y en trabajos especializados (DEC, DBC, Marrero, 1999, Hernández y Samper, 2003). No obstante, no la consideramos para esta investigación porque los informantes sevillanos también la emplearon para el mismo concepto 496. HABICHUElas. En ambas ciudades, además, fue la forma preferida, con un mayor registro de usos que la variante más extendida judías verdes. La distribución fue la siguiente SE: habichuelas 8 / judias verdes 4; LPGC habichuelas 12 / judías verdes 5. Se trata, por tanto, de léxico compartido con distinto porcentaje de uso (Santana, 2011).
} 
En la mayoría de los casos los hablantes cultos se decantaron por la variante más estándar cuando entró en competencia con un regionalismo, cumpliéndose así la tendencia en el sociolecto alto a emplear formas sin marcas diatópicas específicas (Bello, 1847: 165; Trujillo, 1986: 168; Marrero, 1996-1997: 182 y 1999: 393-394). Así sucedió en casi todos los andalucismos registrados entre los hablantes cultos sevillanos y en una buena parte de los canarismos.

Entre las voces locales canarias hay que destacar un grupo significativo que obtuvo un uso mayoritario en la norma culta de LPGC, lo que demuestra su pleno asentamiento en esta comunidad de habla. Fueron los casos de: bochinche, [pan] bizcochado, jugo, papa, millo, pescado salado, pimienta de la puta la madre, papayo, conserva [de membrillo], bizcocho, dulce y aguachirri. En ocasiones el canarismo no fue la respuesta mayoritaria, pero presentó unos porcentajes muy similares a la forma más estandarizada. Así sucedió en arvejas, buche de café y [taza de, agua con] yerbahuerto, en competencia muy equilibrada con guisantes, café corto y yerbabuena, respectivamente.

Dadas las limitaciones de espacio de las que disponemos, nos detenemos de forma más detallada en los andalucismos obtenidos en la norma culta de SE ya señalados arriba. Para mayor información sobre canarismos en la norma culta de LPGC puede consultarse Marrero (1996-1997 y 1999).

Uno de los andalucismos que pervive en la norma culta sevillana es alcaucil, variante local para designar 489. ALCACHOFA. Tanto Alcalá Venceslada (1951) como el TLHA lo documentan como parte del léxico dialectal de esta modalidad de habla. Si bien su representación en el cuestionario 
sevillano no fue mayoritaria $(33.33 \%, 4 / 12)$, hay que destacar la importancia de la pervivencia de esta voz local en el sociolecto alto. Su uso, solo registrado en mujeres, está repartido entre las 3 generaciones. Podría decirse, por tanto, que sigue teniendo cierta vitalidad en la norma sevillana y que la forma estandarizada no ha conseguido desplazarlo del todo.

Bollito obtuvo 1 respuesta para 370. Bocaditos. En el DRAE solo se documenta la voz bollo y, salvo la acepción sexta, que señala como mexicanismo: 'pan en forma de cubilete', no hay otra interpretación similar al valor de esta voz en el léxico culto sevillano. Por su parte, el TLHA dice para bollito que es un 'panecillo que se hace con el resto de la masa'. Pensamos que el uso en la norma culta de SE guarda relación con esta acepción. En efecto, en esta ciudad se empleanen tanto bollo como bollito para designar tipos de pan (el segundo de dimensiones algo más pequeñas). Aunque no se obtuvo respuesta en las encuestas de LPGC, en el español de Canarias la significación de bollo es básicamente un 'dulce con forma redondeada y con un agujero en el centro'(TLEC, DEC). Coincide con lo que en SE podría ser denominado como rosco.

En SE 4 informantes respondieron brócoles para el concepto 495. BRócOLI. El DRAE señala brócol como voz característica de Andalucía, dato que ya aparecía documentado en Alcalá Venceslada (1951). En general se obtuvieron pocas respuestas entre los informantes cultos sevillanos para identificar esta hortaliza, pero se aprecia una preferencia por la variante dialectal $(4 / 6,66.67 \%)$ sobre la forma más general brócoli. El hecho de que el andalucismo se mantenga entre los informantes más jóvenes ( 2 mujeres de la primera 
generación y 1 hombre de la segunda) hace pensar en su continuidad en la comunidad de habla.

Para el concepto 356. LOS CHURRos, 2 informantes sevillanos ( 2 hombres, de la primera y la segunda generación respectivamente) respondieron como segunda opción $c a$ lentitos, frente a la respuesta mayoritaria churros de todos los encuestados, también entre los de LPGC. Tanto el DRAE como las obras lexicográficas más especializadas, TLHA y Alcalá Venceslada (1951), señalan que calentitos es una voz característica de Andalucía para significar 'fruta de sartén', lo que de forma más genérica se denomina churro. En el TLHA se señalan otras voces sinónimas que no fueron empleadas en la norma culta sevillana: cohombro, tallo y tejeringo. Por tanto, calentitos, voz de carácter local, es una muestra de léxico diferencial entre la norma culta de SE y de LPGC, aunque hay que decir que su vitalidad entre los hablantes cultos sevillanos es baja. Cabe destacar, no obstante, que siga perviviendo su uso entre los informantes más jóvenes, aunque puede ser que conozcan la palabra pero que no forme parte de su léxico activo y quizá por eso la han empleado como segunda opción.

La voz chícharos fue empleada por los hablantes cultos sevillanos para responder a los conceptos 501. JUDÍAS BLANCAS, 502. JUDÍAS PINTAS, 503. JUDÍAS NEGRAS, 504. JUDÍAS ROJAS Y 507. guisantes, ChÍcharos, ARVEjAs. ${ }^{8}$ Se trata de la denominación genérica para lo que el DRAE identifica con judías y que define aproximadamente como 'fruto de la planta que

\footnotetext{
${ }^{8}$ Aunque chícharos se documenta entre las voces dialectales canarias (DEC, DBC, Hernández y Samper, 2003), no se registró ninguna respuesta en LPGC.
} 
lleva el mismo nombre y que se caracteriza por tener forma de riñón y alojarse en vainas aplastadas'. En el TLHA se constata la vitalidad de dicha lexía en esta modalidad de habla, en tanto que sirve para denominar distintas variedades de judías.

En las entradas señaladas, no hay unanimidad en lo que respecta a la variación léxica con otras palabras. Si bien chícharos es la respuesta mayoritaria para 501 (8/15, 53.33\%), para el resto de los casos se prefiere judías, variante más estandarizada. La principal alternativa a chícharos en 501 es alubias, mientras que para los otros conceptos es judías.

Los rasgos sociales de los informantes en los que pervive la forma diatópicamente marcada los identifica como mayoritariamente de la tercera generación (3 casos), aunque también hubo 1 mujer del grupo de edad más joven que empleó chícharo para los 4 conceptos, variando la especificación (chícharo blanco, con pintas, negros y colorados). Sin embargo, para la entrada 507. GUISANTES, CHÍcharos, ARVEJAS, donde los informantes sevillanos respondieron preferentemente guisantes, 2 hombres de la segunda generación utilizaron aquí chícharos. Se deduce, por tanto, que no está perfectamente delimitada la significación del vocablo chícharo, que compite en la norma culta sevillana con judía, alubia y, como se observa, también con guisante.

Para el concepto 364. vino BLANCO 3 informantes sevillanos respondieron manzanilla. El resto dijo vino blanco, única respuesta de los encuestados canarios. El DRAE define manzanilla en su octava acepción como 'vino blanco que se cría y elabora en Sanlúcar de Barrameda, en la provincia de Cádiz, España'. El TLHA recoge el término, pero no 
aparece en Alcalá Venceslada (1951), aunque sí términos derivados como manzanillero 'aficionado al vino manzanilla. Por la información lexicográfica consultada pensamos que se trata de un dialectalismo. Esta voz no se utilizó en LPGC porque no es un vino que se tome de forma habitual en aquella zona, todo lo contrario que sucede en SE (muy común en festividades como La Feria de Abril). De hecho, a pesar de que se preguntara por vino blanco de forma genérica, manzanilla apareció entre las respuestas.

El DRAE define pero como 'variedad de manzano, cuyo fruto es más alargado que grueso' y como el 'fruto del pero'. Aunque los académicos no le atribuyen marca diatópica, el vocablo aparece consignado en el TLHA como sinónimo de manzana. La presencia en la norma culta sevillana de este término fue escasa, con solo 1 respuesta de 1 mujer de la segunda generación. Su vitalidad, por tanto, no fue significativa en este sociolecto, donde se prefirió la variante estandarizada manzana.

Picadillo fue la respuesta de 1 mujer de la segunda generación para identificar el 'aliño a base de vegetales, generalmente sin cocer y aliñados'. La respuesta mayoritaria, sin embargo, fue ensalada (única respuesta en LPGC). Cabe decir que ensalada y picadillo no son estrictamente sinónimos. La diferencia estriba en los ingredientes que los componen. Por ejemplo, si lleva lechuga es ensalada, mientras que el picadillo no tiene esta hortaliza. Por tanto, al no haberse especificado en la pregunta que se les hizo a los informantes el tipo de vegetales, se dieron ambas contestaciones. Picadillo es un andalucismo recogido en Alcalá Venceslada (1951). El TLHA documenta su presencia en el ALEA, 
aunque no como respuesta para SE capital. Pensamos que si la pregunta se hubiera planteado de forma más específica se habría obtenido un mayor número de respuestas de este término, que es bastante común en la zona.

Para el concepto 483 la mayoría de los informantes sevillanos respondieron escarola, a excepción de 1 mujer de la primera generación que dijo romanilla. El DRAE no recoge esta acepción, pero sí aparece mencionado en el TLHA y en Alcalá Venceslada (1951) con la significación de 'romana, lechuga'. Se trata, por tanto, de un andalucismo que era previsible, como así ha sucedido, que no utilizaran los informantes canarios. Su pervivencia en la norma culta de SE, a juzgar por los datos, está en franca desventaja con respecto a la voz más estándar escarola.

Para conocer un poco mejor el mantenimiento de voces regionales en la norma culta hemos establecido conexiones con los rasgos sociales de los informantes que las emplearon.

En lo que respecta al sexo, las mujeres sevillanas mostraron un claro predominio de los regionalismos, bien porque fueron las únicas que utilizaron el vocablo (alcaucil, bollitos, pero, picadillo, romanilla) o bien porque registraron más respuestas (brócoles, chícharos). Los hombres sevillanos solo destacaron en el uso exclusivo de la voz calentito, o en el empleo mayoritario de manzanilla. En LPGC, sin embargo, fueron los hombres los que se decantaron por los canarismos en más ocasiones, bien de forma exclusiva (bichillo, bochinche, conduto, garbanzas, jarea, picón, taza de agua) o coincidiendo con mujeres, pero estas con un índice menor de respuestas (arvejas, cafetín, [pollo] compuesto, dulce, 
durazno, enyesques, pescado salado, pimienta de la puta la madre, queque, [taza de, agua con] yerbahuerto). No obstante, también documentamos algunos regionalismos solo empleados por las mujeres sevillanas (beterrada, guanijai, guarapo, pizqueo, quequi, roquillas, tortilla, vuelta) o compartidos con hombres, pero estos con menor índice de frecuencia (bizcocho, bizcochón, jugo, buche de café). El resto de las voces regionales de LPGC tuvieron el mismo número de respuestas en ambos sexos (aguachirri, conserva [de membrillo], [sopa de, caldo de, potaje de] millo, papayo, [huevos] sancochados), en algunos casos porque fueron empleados por todos los informantes ([pan] bizcochado, [caldo de, sopa de] [tortilla de] papas).

En cuanto a la edad, destacamos un ligero incremento de los regionalismos que fueron empleados por los hablantes de la primera generación $(25 / 45,55,56 \%)$, que es previsible que continúen en su correspondiente comunidad de habla (SE: alcaucil, brócoles, calentitos, chícharos, romanilla; LPGC: aguachirri, arvejas, [pan] bizcochado, bizcocho, buche de café, conserva [de membrillo], dulce, durazno, guarapo, jarea, jugo, [sopa de, caldo de, potaje de] millo, [caldo de, sopa de] [tortilla de] papas, papayo, pescado salado, picón, pimienta de la puta la madre, queque, [taza de, agua con] yerbahuerto, [huevos] sancochados). Por su parte, los casos registrados solo en la tercera generación (SE: manzanilla; LPGC: quequi, garbanzas, rosquillas, tortilla) o no utilizados por los informantes más jóvenes (SE: bollitos, pero, picadillo; LPGC: beterrada, bichillo, bizcochón, bochinche, cafetín, [pollo] compuesto, conduto, enyesques, guanijai, pizqueo, taza de agua, vuelta) muestran menor 
indicio de continuidad para el valor con el que han sido empleados.

\subsection{Palabras empleadas en ambas ciudades pero con sentidos diferentes}

Tanta información aportan para nuestros fines las palabras distintas de SE y de LPGC como aquellas que coinciden en ambas áreas, pero que se emplean con peculiaridades que las singularizan en cada una de ellas. A este respecto, hubo voces compartidas, pero con distinta significación: bizcocho, manzanilla y tortilla.

Bizcocho obtuvo respuesta en SE y en LPGC, pero para conceptos distintos. En concreto, los informantes sevillanos emplearon bizcocho con el sentido de la primera acepción que señala el DRAE, 'dulce blando y esponjoso, hecho generalmente con harina, huevos y azúcar, que se cuece en el horno', que se corresponde de forma aproximada con el concepto 564. BizcoсHO 9 y los informantes canarios la emplearon preferentemente como respuesta a 355. PAN TOSTADO, aunque también para responder a 564. BizCOCHO Y a 568. TORTA.

\footnotetext{
${ }^{9}$ A pesar de que la realidad por la que se pregunta es muy común entre los sevillanos, resulta extraño que 5 informantes no contestaran. Esto se debe a una interferencia que se produjo entre el cuestionario seguido como modelo, el diseñado por el PILEI, y el que se pasó en SE. Como indica Díaz (1997: 455), en un primer momento los informantes no identificaban bizcocho con la definición propuesta en el cuestionario ("pan dulce sin levadura, que se cuece dos veces para que dure"), por lo que hubo que añadir otra ("masa compuesta de harina, huevos, azúcar y levadura que se cuece al horno”), que sí reconocieron los encuestados.
} 
En lo que respecta al uso de bizcocho como sinónimo de pan tostado, se alude a la definición que da el DRAE en su segunda acepción 'pan sin levadura, que se cocía por segunda vez para que se perdiese la humedad y durase mucho tiempo. Como ya señalamos para bizcochado, este es un uso arcaico que guarda relación con el significado etimológico de la palabra. Aparece consignado en el TLEC, donde se alude a una costumbre de los campesinos para que la masa se conserve durante más tiempo. Su aparición también en el Atlas lingüistico y etnográfico de las Islas Canarias [ALEICan] refleja el asentamiento de esta voz en las Islas. Por su parte, Marrero (1999: 375) cataloga bizcocho con este valor como canarismo de origen patrimonial empleado por los hablantes cultos de LPGC.

Aunque el número de respuestas de bizcocho no fue elevado, un total de 5, resulta significativo que estén repartidas entre todas las generaciones (1 mujer y 1 hombre de la primera generación, 1 mujer de la segunda y 1 mujer y 1 hombre de la tercera generación), lo que demuestra su pervivencia en la norma culta canaria. Por su parte, pan bizcochado es una variante plenamente asentada en LPGC, pues obtuvo respuesta entre todos los informantes, principalmente como única o como primera respuesta. Solo en 2 casos fue la segunda opción (2 mujeres de la segunda y de la tercera generación respectivamente, ambas con primera respuesta pan tostado) y en 1 caso como tercera opción (hombre de la primera generación), habiendo respondido bizcocho en primer lugar y pan tostado en segundo. Los jóvenes mantienen bizcochado como única respuesta, síntoma de la pervivencia de la lexía en esta comunidad de habla. 
Es interesante señalar también que en LPGC bizcocho, como respuesta al concepto 564, entró en competencia en esta ciudad con otra variante léxica de carácter local: queque/quequi, ${ }^{10}$ considerada canarismo (TLEC, DEC, DBC, Marrero, 1999: 375). Los datos de las encuestas nos muestran que bizcocho y queque están en clara competencia en la norma culta de la capital canaria, con una rentabilidad muy equilibrada: bizcocho $(8 / 18,44.44 \%)$ y queque $(6 / 18$, $33.33 \%)$. En ambos casos, casi todos los informantes emplearon una $\mathrm{u}$ otra variante como única o como primera opción y con representación en todos los grupos etarios. No parece, de momento, que el canarismo haya perdido la batalla con respecto al empuje de la forma más estandarizada. Cabe señalar que se empleó una tercera forma de carácter local, bizcochón, pero en este caso con menor vitalidad entre los hablantes cultos canarios $(3 / 18,16.67 \%)$. Solo 1 persona de la tercera generación contestó bizcochón sin hacer uso de otra variante sinonímica, las otras 2 , de la segunda generación, lo emplearon como tercera opción (junto a bizcocho y queque), lo que confirma que es un vocablo con una menor pervivencia en la norma culta de LPGC y que, además, al no ser empleado por los informantes más jóvenes, podría presentar tendencia a la desaparición.

Manzanilla se utilizó en SE y en LPGC como respuesta para el concepto 625. INFUSIÓN DE MANZANILLA. Sin embargo, como ya se dijo, en SE 3 informantes (de la tercera generación) respondieron también esta palabra para el concepto 364. vinO BLANCO. El uso de esta forma léxica

${ }^{10}$ Variantes de pronunciación. Queque es más frecuente. 
puede considerarse, por tanto, un ejemplo de léxico diferencial entre las ciudades de SE y LPGC.

Si bien la voz tortilla es empleada tanto en SE como en LPGC para los conceptos 413. HUEVOS EN TORTILla; 414. OMELETTE, TORTILLA A LA FRANCESA; Y 415. TORTILLA DE PATATAS, en LPGC se empleó con otra significación. Resulta interesante la utilización, aunque escasa, de tortilla en la ciudad canaria para el concepto 547. TORRIJAS, TORREJAS, con una acepción característica y que no es compartida con los sevillanos. Se emplea, al menos en Gran Canaria, para denominar un alimento dulce compuesto por una 'masa compuesta por harina, huevos y azúcar, que se fríe, y que puede llevar distintos rellenos: cabello de ángel, batata, etc.' Las tortillas se hacen para fiestas específicas como las Navidades, los Carnavales o el día de la Comunidad Autónoma de Canarias. Aparecen con esta denominación en los libros de gastronomía de la zona.

En realidad tortilla no es una respuesta esperable para el concepto 547, porque al informante se le pregunta por la "rebanada de pan remojada en vino o leche y bañada después en miel o almíbar", es decir, lo que se conoce de forma común en España con la denominación de torrija (el DRAE da una definición en términos muy similares), que es la única respuesta de los informantes sevillanos y la mayoritaria en LPGC. De hecho, la mujer que responde tortilla (de la tercera generación) lo hace como segunda opción, la primera fue torrija. La razón de este uso léxico parece estar en que, como se explica en el TLEC, las torrijas eran en Gran Canaria un tipo de tortilla (en el sentido que hemos descrito arriba), bañadas en miel, 
que se hacían en el sur de la isla durante los Carnavales o por ocasión de una boda. Es decir, en el sur de la isla se llamaban tortillas lo que habitualmente denominamos torrijas. Solo una informante de edad más avanzada recuerda esta significación.

El DRAE no recoge las acepciones de tortilla que aquí estamos señalando para el español de Canarias. Solo la hemos documentado en el $D E C$, como una forma específica de denominar a las tortillas de carnaval, y se definen como localismo que se da en Gran Canaria y en La Gomera. Hay que considerarlo, por tanto, una diferencia con respecto al léxico culto de SE, una pervivencia de una denominación característica de la isla de Gran Canaria, aunque al no haber una pregunta específica para este tipo de alimento, obtuvo poca presencia en las encuestas.

\subsection{Variaciones entre una voz estándar y un localismo con distinta distribución en ambas ciudades}

Un tercer grupo será el de la alternancia entre un término estándar y una variante más local, variación que puede ayudar a perfilar los usos cultos de cada una de las zonas. Concretamente estudiaremos la convivencia de las parejas maíz-millo y patata-papa.

Frente a la voz generalizada maíz, única respuesta en SE en una lexía compleja (sopa de maíz), en la norma culta de LPGC dicha forma alternó con el regionalismo de origen portugués millo. Esta variante presenta mayor vitalidad entre los informantes canarios que la forma estándar, con un 
porcentaje del 76.47\% de usos (13/17). Nuestros datos coinciden con los de Hernández y Samper (2003: 347), lo que demuestra la vitalidad que tiene este término, que pervive en la norma culta y en las generaciones jóvenes. De hecho, de los 3 informantes que emplearon la variante más generalizada, solo 1 de ellos es de la primera generación.

Y por último nos ocupamos de la variación papas-patatas. ${ }^{11}$ Estas voces fueron empleadas en ambas ciudades, aunque con índices de frecuencia distintos. En SE es habitual la convivencia de las 2 variantes, tal y como se observa en la norma culta. Concretamente, los hablantes del sociolecto alto prefirieron la forma patata: fue la única repuesta para 389, sopa de patata, y la mayoritaria para 415 , tortilla de patatas, con un porcentaje del 71.43\% (5/7). Tortilla de papas solo fue utilizada por 2 informantes de la tercera generación. Por su parte, en LPGC es previsible que se emplee de forma exclusiva papa, aunque no es eso lo que documentamos en la norma culta. En concreto, hubo 1 contestación para 389. como SOPA DE PAPAS (mujer de la tercera generación) y 2 para 415, de toRTILla de PATATAs (2 mujeres de la primera y la tercera generación respectivamente). En este caso son las féminas las que dan muestra del proceso de estandarización, aunque los datos apuntan a que la variante local papa es la que mayor presencia tiene en la norma culta de LPGC. Nuestros resultados coinciden con los de Hernández y Samper (2003: 347), quienes consignaron las 2 variantes en el léxico disponible de estudiantes preuniversitarios, aunque

${ }^{11}$ En el cuestionario del PILEI también se utilizan las dos variantes. 
el americanismo papa obtuvo una frecuencia mucho más significativa y apareció en más contextos.

\section{Conclusiones}

El estudio contrastivo del léxico de la alimentación en la norma culta de SE y de LPGC ha puesto de manifiesto particularidades en cada una de estas ciudades que contribuyen a acentuar su identidad. Las diferencias se dieron en tres bloques: palabras distintas que solo se emplearon en una zona; uso de una misma palabra pero con sentidos diferentes; $y$ la distinta distribución de una variante estandarizada y un localismo.

En el primer caso, aunque hubo algunos conceptos desconocidos para los hablantes cultos sevillanos (berro, cilantro, gofio y [alimento] sancochado), la mayoría de las veces se trataba de formas distintas de denominar una realidad que era reconocida por los informantes de ambas ciudades. No siempre fueron lexías totalmente diferentes, sino que hubo bases léxicas compartidas que generaron variaciones de pronunciación-escritura, morfológicas de género o de derivación apreciativa en las áreas estudiadas.

En las variantes con distinta pronunciación-escritura, champagne fue la forma preferida por los hablantes cultos sevillanos, por encima de champán, que fue la única empleada en LPGC. Destacamos además la vitalidad de restaurán frente a restaurante en la capital canaria, principalmente entre las mujeres de las generaciones más jóvenes. 
En cuanto a la variación de género, tanto papayo en LPGC como tostada en SE fueron denominaciones plenamente asentadas para sus correspondientes conceptos, con más vitalidad que otras lexías más estándares: papaya y [pan] tostado, respectivamente.

En lo que respecta al diminutivo, cuando se empleó como sufijo derivativo, habría que comprobar si las tendencias observadas en el campo de la alimentación se mantienen en otros campos semánticos: mayor presencia del diminutivo en la capital andaluza y tendencia a la distribución -ito para SE e -illo para LPGC. De ser así hablaríamos de una marca diferenciadora del léxico culto de ambas ciudades. Por lo general las variantes con este sufijo obtuvieron pocas respuestas para cada uno de sus conceptos y se apreció un ligero aumento entre las mujeres.

Cuando el diminutivo estaba lexicalizado se dieron casos de regionalismos que, siguiendo la tendencia generalizada en el corpus, tuvieron menor aceptación que otras formas estandarizadas más comunes: canapés-bollitos, churros-calentitos, lechuga-picadillo, carne rellena-redondilla, escarola-romanilla, infusión-agüita, solomillo-bichillo y pastas-rosquillas.

En los casos en los que las diferencias se debieron al empleo de regionalismos en una de las ciudades, de forma general se prefirió la variante más estándar, constatándose así la tendencia a un uso lingüístico más homogéneo y menos marcado en el sociolecto alto. No obstante, esta característica no se aplica a un grupo de formas locales empleadas mayoritariamente (o con porcentajes muy similares a los de la variante del español general) por los hablantes cultos canarios, con lo que cabe decir que están asentados en esta comunidad 
de habla y, además, en este nivel sociocultural (aguachirri, arvejas, bizcocho, bochinche, buche de café, conserva [de membrillo], dulce, millo, [pan] bizcochado, [caldo de, sopa de] [tortilla de] papas, papayo, pescado salado, pimienta de la puta la madre y [taza de, agua con] yerbahuerto). Solo documentamos 1 caso en la norma culta de SE (brócoles más usado que brócoli). En cuanto a la distribución sociocultural, la variable sexo mostró resultados distintos en una y otra ciudad: en SE las mujeres hicieron mayor uso de estas voces, y en LPGC fueron los hombres los más aventajados. En lo que respecta a la edad, hubo un número ligeramente superior de voces locales que fueron empleadas al menos en 1 ocasión por informantes de la primera generación $(25 / 45,55,56 \%)$, lo que hace pensar en su pervivencia en la norma culta de sus correspondientes comunidades de habla.

El segundo bloque de palabras que estudiamos, las empleadas en las dos ciudades pero con sentidos distintos, nos llevó a conocer las particularidades de bizcocho (con el sentido de 'pan tostado'; y para significar 'masa dulce' en estrecha competencia con queque, variante más local) y tortilla (un tipo de torrija) en la norma culta de LPGC; y de manzanilla (un tipo de vino blanco) en la norma culta de SE.

Y en tercer lugar, la distribución de las parejas maízmillo y patata-papa reveló una preferencia en LPGC por las variantes locales millo y papa, frente a SE, donde solo se usó maíz y donde se respondió mayoritariamente patata.

Los resultados obtenidos en esta investigación indican que a pesar de la tendencia a los usos lingüísticos homogéneos y sin marca diatópica del sociolecto alto, se advierten para el campo de la alimentación particularidades léxicas 
que aportan singularidad a la norma culta de SE y de LPGC. La comparación de otros campos semánticos nos ayudará a tener una visión más acertada de las tendencias que aquí se han apuntado y contribuirá a perfilar las diferencias y las semejanzas entre ambas ciudades.

\section{Bibliografía}

Academia Canaria de la Lengua (2010), Diccionario básico de canarismos [DBC], Las Palmas de Gran Canaria, Academia Canaria de la Lengua-Gobierno de Canarias. Alcalá Venceslada, A. ([1951] 1998), Vocabulario andaluz, Jaén, Servicio de Publicaciones de la Universidad de Jaén.

Alvar, M. (1975-1978), Atlas lingüistico y etnográfico de las Islas Canarias [ALEICan], Las Palmas de Gran Canaria, Publicaciones del Excmo. Cabildo Insular.

Alvar, M., A. Llorente y G. Salvador (1961-1973), Atlas lingüistico y etnográfico de Andalucía [ALEA], Granada, Universidad de Granada / Consejo Superior de Investigaciones Científicas.

Alvar Ezquerra, M. (2000), Tesoro léxico de las hablas andaluzas [TLHA], Madrid, Arco Libros.

Bello, A. ([1847] 1988): Gramática de la lengua castellana destinada al uso de los americanos, estudio y edición de R. Trujillo, Madrid, Arco Libros.

Carbonero Cano, P. (dir.) y A. Ortiz Torres (ed.) (2005), Léxico del habla culta de Sevilla. Sociolingüística Andaluza 14, Sevilla, Publicaciones de la Universidad. 
Corrales, C. y D. Corbella (2009), Diccionario ejemplificado de canarismos [DEC], La Laguna (Tenerife), Instituto de Estudios Canarios.

Corrales, C., D. Corbella y Ma á. Álvarez (1992), Tesoro lexicográfico del español de Canarias [TLEC], Madrid, Real Academia Española.

DíAz, R. (1997), "Una investigación sobre el léxico andaluz: métodos y resultados en el estudio léxico del habla de Sevilla”, en A. Narbona y M. Ropero (eds.), El habla andaluza. Actas del Congreso del Habla Andaluza. Sevilla, Excmo. Ayuntamiento de Sevilla-Universidad de Sevilla, pp. 453-460.

Fernández Sevilla, J. (1981), “Andalucía y Canarias: relaciones léxicas", en M. Alvar (coord.), I Simposio Internacional de la Lengua Española, Las Palmas de Gran Canaria, Excmo. Cabildo Insular de Gran Canaria, pp. 71-126.

García Mouton, P. (1999), Cómo hablan las mujeres, Madrid, Arco Libros.

Hernández, C. y J. A. Samper (1999), “El campo léxico de la enseñanza en la norma lingüística culta de España y América”, en J. A. Samper y M. Troya (coords.), Actas del XI Congreso de la ALFAL, vol. 2, Las Palmas de Gran Canaria, Universidad de Las Palmas de Gran Canaria, pp. 1067-1078.

(2003), "Los dialectalismos en el léxico disponible de Las Palmas de Gran Canaria. Análisis de un centro de interés", en F. Moreno Fernández, F. Gimeno, J. A. Samper, $M^{a}$ L. Gutiérrez Araus, $M^{a}$ Vaquero y C. Hernández (eds.), Lengua, variación y contexto. Estudios dedi- 
cados a Humberto López Morales, vol. 1, Madrid, Arco Libros, pp. 339-354.

López Morales, H. (1992), "Muestra del léxico panantillano: el cuerpo humano", en Scripta Philologica in honorem Juan M. Lope Blanch, vol. 2, México, Universidad Nacional Autónoma de México, pp. 593-625.

LunA, E. (1997), "Muestra de léxico panhispánico: el cuerpo humano", en Anuario de Letras, XXXV, pp. 313-333. (2005), "Notas a propósito de algunas diferencias léxicas en el léxico culto de México y Bogotá", en M. Lozano Ramírez (coord.), Homenaje a José Joaquín Montes Giraldo. Estudios de dialectología, lexicografía, lingüistica general, etnolingüística e historia cultural, Bogotá, Instituto Caro y Cuervo, pp. 452-457.

- (2007), "Coincidencias y discrepancias léxicas en cuatro ciudades de habla hispana", Revista de Filología de la Universidad de La Laguna, 25, pp. 353-360.

Marrero, V. (1996-1997), "Canarismos en la norma lingüística culta de Las Palmas de Gran Canaria”, Philologica Canariensia: Revista de Filología de la Universidad de Las Palmas de Gran Canaria, 2-3, pp. 163-184.

- (1999), La estructura del léxico en la norma lingüística culta de Las Palmas de Gran Canaria, Las Palmas de Gran Canaria, Servicio de Publicaciones de la Universidad.

Moreno Fernández, F. (1991), "Relaciones léxicas entre Colombia, Andalucía y Canarias (agricultura y ganadería)", en El español de América. Actas del III congreso Internacional de El Español de América, vol. 2, Junta de Castilla León, pp. 815-826. 
Programa Interamericano De Lingüística y EnseÑANZA DE IDIOMAS [PILEI] (1972), Cuestionario para el estudio coordinado de la norma lingüística culta de las principales ciudades de Iberoamérica y de la Península Ibérica, vol. 3, Léxico, Madrid, Consejo Superior de Investigaciones Científicas.

Real Academia Española (2001), Diccionario de la lengua española [DRAE], 23a . ed., Madrid, Espasa Calpe.

SAlvador, F. (1992), "El campo léxico de la enseñanza en el habla culta de México y de Granada”, en Scripta Philologica in honorem J.M. Lope Blanch, vol. 2, México, Universidad Nacional Autónoma de México, pp. 231-246. SAmper, José Antonio (dir.) (1998), Léxico del habla culta de Las Palmas de Gran Canaria, Las Palmas de Gran Canaria, Publicaciones de la Universidad.

SAntANA, J. (2011), "El campo de la alimentación en la norma lingüística culta de Sevilla y de Las Palmas de Gran Canaria: léxico compartido", en Y. Congosto y E. Méndez (eds.), Variación lingüística y contacto de lenguas en el mundo hispánico, Fráncfort / Madrid, Iberoamericana / Vervuert, pp. 679-696.

(2014), "El uso del diminutivo en el campo de la alimentación: estudio en el léxico del habla culta de Sevilla y de Las Palmas de Gran Canaria", comunicación pronunciada en las Jornadas Internacionales sobre la Variación en Español en Homenaje al Profesor Dr. D. Manuel Ariza Vigera, Sevilla, Universidad de Sevilla (7-9 de abril de 2014).

Trujillo, R. (1986), "Hablar canario”, en M. Alvar (coord.), Lenguas peninsulares y proyección hispánica, Madrid, 
Fundación Friedrich Ebert / Instituto de Cooperación Iberoamericana, pp. 163-174.

Valencia, A. (1984), "Las comidas de un día ordinario. Análisis de un campo léxico", Anuario de Letras, XXII, pp. 235-250. 
